\title{
Role of Density Profiles for the Nonlinear Propagation of Intense Laser Beam through Plasma Channel
}

\author{
Sonu Sen, ${ }^{1}$ Meenu Asthana Varshney, ${ }^{2}$ and Dinesh Varshney ${ }^{3}$ \\ ${ }^{1}$ Department of Engineering Physics, Indore Institute of Science and Technology, Indore 453331, India \\ ${ }^{2}$ Department of Physics, M. B. Khalsa College, Indore 452002, India \\ ${ }^{3}$ School of Physics, Vigyan Bhawan, Devi Ahilya University, Khandwa Road Campus, Indore 452001, India \\ Correspondence should be addressed to Sonu Sen; ssen.plasma@gmail.com
}

Received 12 November 2013; Revised 3 February 2014; Accepted 17 February 2014; Published 23 March 2014

Academic Editor: Partha P. Banerjee

Copyright (c) 2014 Sonu Sen et al. This is an open access article distributed under the Creative Commons Attribution License, which permits unrestricted use, distribution, and reproduction in any medium, provided the original work is properly cited.

\begin{abstract}
In this work role of density profiles for the nonlinear propagation of intense laser beam through plasma channel is analyzed. By employing the expression for the dielectric function of different density profile plasma, a differential equation for beamwidth parameter is derived under WKB and paraxial approximation. The laser induces modifications of the dielectric function through nonlinearities. It is found that density profiles play vital role in laser-plasma interaction studies. To have numerical appreciation of the results the propagation equation for plasma is solved using the fourth order Runge-Kutta method for the initial plane wave front of the beam, using boundary conditions. The spot size of the laser beam decreases as the beam penetrates into the plasma and significantly adds self-focusing in plasma. This causes the laser beam to become more focused by reduction of diffraction effect, which is an important phenomenon in inertial confinement fusion and also for the understanding of self-focusing of laser pulses. Numerical computations are presented and discussed in the form of graphs for typical parameters of laser-plasma interaction.
\end{abstract}

\section{Introduction}

There has been considerable interest in the interaction of laser beam with plasma. Among the nonlinear optical effects, the self-interaction of intense laser beam occupies a significant place. The interaction of intense laser beam with plasma modifies the dielectric function of the medium. Moreover, the dielectric constant of the plasma depends on amplitude of laser beam, which further changes the propagation characteristics of the laser beam. Laser beam propagating in plasma channel can create its own waveguide in which geometric and diffraction divergence are removed and beam is self-focused. Self-focusing of laser beams is an important nonlinear process in laser produced plasma and laser based charged particle accelerators. For ultrafast laser pulses lasting the order of a picoseconds or less, the drift velocity of electrons in a plasma can be comparable to the velocity of light, causing a significant increase in the mass of the electron and consequently in the effective dielectric constant of the plasma. The nonlinearity in the dielectric constant arises on account of relativistic variation of mass for arbitrary magnitude of intensity and the density perturbations.

With the recent advancements in laser technology beam power can be a thousand times larger than the critical value. High power lasers are capable of achieving very high electric field and thus plasma can sustain extremely intense electric fields in which the plasma electron oscillates at relativistic velocities and, hence, the relativistic effect observed at such a high intensity of beam. For short laser pulses ion motion can be disregarded. In this case the nonlinearity of the medium due to propagation of the beam is determined by the relativistic motion of electrons. The ponderomotive nonlinearity plays an important role in self-focusing of laser beam due to expulsion of the electrons from the focal spot. A ponderomotive force attracts a particle regardless of its charge to low intensity field areas and repels it from high field areas.

The ponderomotive force associated with an intense laser beam expels electrons radially and can lead to cavitation in plasma. At high intensity these two factors, that is, relativistic effect and ponderomotive nonlinearity, contribute 
to focusing on a femtosecond time scale. The relativistic mass increase of the oscillating electrons leads to a decrease in the plasma frequency and thus a local increase in the refractive index. The ponderomotive expulsion of electrons from the beam path such that the ponderomotive force is balanced by the electrostatic field set up between the displacement electrons and the ions that remains on axis. These factors lead to a positive focusing effect that becomes stronger as the laser beam decreases in diameter and becomes more intense.

Relativistic interaction of a laser beam with plasma has been studied experimentally and theoretically by many authors in the past decades. High intensity laser-plasma interactions are of much current interest because of their application to laser-plasma accelerators $[1,2]$, laser-plasma based harmonic generation [3, 4], X-ray lasers [5], and laser-driven inertial confinement fusion schemes $[6,7]$. In these applications, one needs the laser beam to propagate over several Rayleigh lengths while preserving an efficient interaction with the plasmas. Therefore, self-focusing of a high-intensity laser beam and its propagation up to as much possible Rayleigh length in plasma is a very important research issue nowadays [8-14].

When the beam power corresponds to the irradiance in the range where the dependence of the dielectric constant on the irradiance is pronounced, the radial variation of the dielectric constant may be strong enough to cause convergence, which overcomes the divergence due to diffraction. As the beam converges to a minimum level and again diverges due to dependence of the dielectric constant on irradiance of a Gaussian laser beam; this trend continues until the beam acquires its original width, after which the cycle gets repeated. This situation corresponds to oscillatory convergence or selffocusing. Beyond the focus, the nonlinear refraction starts weakening and the spot size of the laser increases, showing oscillatory behavior with the distance of propagation.

To overcome the diffraction and the successive high amplitude oscillation of the spot size, increasing plasma density gradients are introduced. The combined effect of ponderomotive and relativistic nonlinearities in the presence of density profiles are significant for the penetration of superintense laser pulses into plasmas especially regarding self-focusing and self-channelling phenomena. A preformed plasma density profile can prevent the beam diffraction and allows the propagation of an intense laser beam with long distance, and this is beneficial to the abovementioned applications.

In view of this in the present paper we have made an analytical investigation followed by numerical calculations to study relativistic ponderomotive self-focusing of a laser beam in inhomogeneous plasma with different density profiles. Based on WKB and paraxial theory, the critical curves are drawn and laser spot size evolution is analysed for inhomogeneous plasma. Numerical results and discussions are made next supported by figures.

\section{Ponderomotive Self-Channeling}

The laser pulses generating relativistic intensities are tightly focused-quite in contrast with electrons in plane waves.
Averaging the equation of motion of an electron in such an inhomogeneous field over the fast laser oscillations one arrives in the case of low laser field strength, at the description of a force acting on the electron. This force is called ponderomotive force and is directed along the gradient of the laser intensity. For a Gaussian beam profile this means that electrons are expelled from the optical axis.

Consider the propagation of an intense Gaussian laser beam through a cold plasma of electron density $n_{0}$, propagating along $z$-direction. The amplitude of the electric vector $\mathbf{E}$ in cylindrical coordinate can be expressed as

$$
\mathbf{E}=\widehat{x} A(r, z, t) \exp [-i(\omega t-k z)]
$$

where

$$
\begin{gathered}
A^{2}=A_{00}^{2} g(t) \exp \left(\frac{-r^{2}}{r_{0}^{2}}\right), \\
k(z)=\left(\frac{\omega}{c}\right) \varepsilon_{0}^{1 / 2}, \\
\omega_{p}(z)=\left(\frac{4 \pi n_{0}(z) e^{2}}{m}\right)^{1 / 2}
\end{gathered}
$$

is the electron plasma frequency, $r_{0}$ is the initial radius of the beam, $\varepsilon_{0}^{\prime}$ is the plasma dielectric constant and choosing steppulse function $g(t)=1$ for $t>0$ and $g(t)=0$ otherwise, " $e$ " and " $m$ " are the electronic charge and mass, respectively, and " $c$ " is the velocity of light. The index of refraction or dielectric function $\varepsilon$ can be written as

$$
\varepsilon(r)=\left[1-\left(\frac{\omega_{p}^{2}}{\omega^{2}}\right)\left(\frac{n_{e}(r)}{n_{0} \gamma(r)}\right)\right],
$$

here, $n_{e}(r)$ is the radial distribution of electron density, $\gamma(r)$ is the relativistic factor associated with the electron motion transverse to the laser propagation, and $\omega$ is the laser frequency. The dielectric function $\varepsilon$ has dependence on irradiance $E E^{*}$ of a Gaussian beam and hence $\varepsilon$ is the function of $r^{2}$; therefore in the paraxial approximation $\varepsilon$ can be expressed in powers of $r^{2}$. It will be seen from this that an on-axis maximum can be created through modification of the radial profile of $\gamma$ and/or $n_{e}$.

Considering the intensity dependence of electron mass, the relativistic ponderomotive force produced due to laser beam is

$$
\mathbf{F}_{p}=e \nabla \phi_{p}
$$

where

$$
\phi_{p}=-\left(\frac{m c^{2}}{e}\right)(\gamma-1)
$$

is the ponderomotive potential with relativistic factor $\gamma=$ $\left[1+a^{2} / 2\right]^{1 / 2}$ and $a=(e|A| / m \omega c)$. At the front of the laser pulse ponderomotive force has axial and radial components. The radial ponderomotive force pushes the electrons radially 
outward, on the time scale of a radial space charge field $\mathbf{E}_{s}=$ $\left(-\nabla \phi_{s}\right)$. Using Poisson's equation,

$$
\nabla^{2} \phi_{s}=4 \pi e\left(n_{e}-n_{0}\right) .
$$

For $\omega_{p} \tau>1$, assuming a quasisteady-state with $\phi_{s}=-\phi_{p}$ and using (4) in (6), we obtain modified electron density as

$$
n_{e}(z)=n_{0}(z)\left[1+\frac{c^{2}}{\omega_{p}^{2}(z)} \nabla_{\perp}^{2} \gamma\right]
$$

Equation (7) is valid in those regions of " $r$ " where the second term in the square bracket is greater than -1 ; otherwise $n_{e}=$ 0 . Thus there is complete electron evacuation in region where

$$
\left[1+\left(\frac{c^{2}}{\omega_{p}^{2}(z)}\right) \nabla_{\perp}^{2} \gamma\right]<0 .
$$

Assume that the pulse propagates without changing shape and employing a Gaussian constant shape ansatz for amplitude as $[15,16]$

$$
q^{2}=\frac{q_{0}^{2}}{f^{2}} \exp \left(\frac{-r^{2}}{r_{0}^{2} f^{2}}\right)
$$

where $f$ is the dimensionless beamwidth parameter which is unity at $z=0, r^{2}=\left(x^{2}+y^{2}\right)$ is the radial component in cylindrical coordinate system, $r_{0}$ is the initial beamwidth, and $q_{0} \cong\left(e A_{00} / m \omega c\right)$ is the axial amplitude of the laser giving the modified electron density as

$$
n_{e}(z)=n_{0}\left[1-\frac{c^{2}}{\omega_{p}^{2} r_{0}^{2} f^{2}} \frac{q^{2}}{\gamma} \times\left(1-\frac{r^{2}}{r_{0}^{2} f^{2}} \frac{1+q^{2} / 4}{\gamma^{2}}\right)\right] .
$$

Using (10) in (3) and making Taylor expansion of dielectric function in the radial direction for arbitrary large nonlinearity under paraxial approximation can be written as

$$
\varepsilon=\varepsilon_{0}^{\prime}-\frac{\varepsilon_{1} r^{2}}{r_{0}^{2}}
$$

where

$$
\begin{gathered}
\varepsilon_{0}^{\prime}=1-\frac{\omega_{p}^{2} / \omega^{2}}{\gamma_{0}^{\prime}}\left(1-\frac{c^{2}}{\omega_{p}^{2} r_{0}^{2} f^{2}} \frac{q_{0}^{2} / f^{2}}{\gamma_{0}^{\prime}}\right), \\
\varepsilon_{1}=\frac{\omega_{p}^{2}}{4 \omega^{2}} \frac{q_{0}^{2} / f^{4}}{\gamma_{0}^{\prime 3}}\left(1+\frac{c^{2}}{\omega_{p}^{2} r_{0}^{2} f^{2}} \frac{8+q_{0}^{2} / f^{2}}{\gamma_{0}^{\prime}}\right), \\
\gamma_{0}^{\prime}=\left(1+\frac{q_{0}^{2}}{2 f^{2}}\right)^{1 / 2} .
\end{gathered}
$$

\section{Laser Spot Size Evolution}

The wave equation for the propagation of the laser is given as

$$
\frac{\partial^{2} \mathbf{E}}{\partial z^{2}}+\nabla_{\perp}^{2} \mathbf{E}+\frac{\omega^{2}}{c^{2}} \varepsilon(r, z) \mathbf{E}=0
$$

Using the WKB approximation in inhomogeneous plasma the starting point is a solution of the form

$$
E(r, z)=A(r, z) \exp \left[-\int_{0}^{z} i k(z) d z\right]
$$

where $A(r, z)$ is the slowly varying complex amplitude of the electric field. Substituting for $E(r, z)$ from (14) in (13) and neglecting the second order derivatives, the envelope equation is obtained as

$$
-2 i k \frac{\partial A}{\partial z}+\nabla_{\perp}^{2} A+\left(\frac{\omega^{2}}{c^{2}} \varepsilon-k^{2}\right) A=0
$$

Here $\varepsilon$ is dielectric constant under relativistic mass and ponderomotive nonlinearity which is function of irradiance of a Gaussian beam and hence function of $r^{2}$. Using the effective dielectric function given by (11), the envelope equation (15) can be rewritten as

$$
\begin{aligned}
-2 i k & \frac{\partial A}{\partial z}-i A \frac{\partial k}{\partial z}-k^{2} A+\frac{1}{r} \frac{\partial A}{\partial r}+\frac{\partial^{2} A}{\partial r^{2}} \\
+\frac{\omega^{2}}{c^{2}}\left(\varepsilon_{0}^{\prime}-\varepsilon_{1} \frac{r^{2}}{r_{0}^{2}}\right) A & =0 .
\end{aligned}
$$

For nearly spherical wave front (a valid assumption in the paraxial approximation), the complex amplitude $A(r, z)$ may be expressed as

$$
A(r, z)=A_{r}(r, z) \exp [-i k S(r, z)]
$$

where $A_{r}$ and $S$ are real and the eikonal $S$ is given by

$$
S=\frac{r^{2}}{2}\left(\frac{1}{f} \frac{d f}{d z}\right)+\varphi(z)
$$

where $(1 / f)(d f / d z)$ represents the inverse of the radius of curvature of the wave front. Substituting for $A(r, z)$ and $S(r, z)$ from (17) and (18) in (16) and equating the real and imaginary parts on both sides of the resulting equation, one obtains

$$
\begin{gathered}
2\left(\frac{\partial S}{\partial z}\right)+\left(\frac{\partial S}{\partial r}\right)^{2}+\frac{2 S}{k(z)}\left(\frac{\partial k}{\partial z}\right) \\
=\frac{1}{k^{2} A_{r}}\left(\frac{\partial^{2} A_{r}}{\partial r^{2}}+\frac{1}{r} \frac{\partial A_{r}}{\partial r}\right)-\frac{\varepsilon_{1}}{\varepsilon_{0}^{\prime}} \frac{r^{2}}{r_{0}^{2}} \\
\frac{\partial A_{r}^{2}}{\partial z^{2}}+\frac{\partial S}{\partial r} \frac{\partial A_{r}^{2}}{\partial r}+A_{r}^{2}\left(\frac{\partial^{2} S}{\partial r^{2}}+\frac{1}{r} \frac{\partial S}{\partial r}\right)=-A_{r}^{2} \frac{1}{k}\left(\frac{\partial k}{\partial z}\right) .
\end{gathered}
$$

The solution of (19) is

$$
A_{r}^{2}=\frac{A_{00}^{2}}{f^{2}} \exp \left(\frac{-r^{2}}{r_{0}^{2} f^{2}}\right) .
$$

Now substitute for $A_{r}$ from (21) in (20) and making use of the paraxial ray approximation, that is, $\left[\left(r / r_{0} f\right)^{4} \ll 1\right]$. Equating 
the coefficients of $r^{2}$ on both sides, we get resulting equation governing the beamwidth parameter as

$$
\frac{1}{f}\left(\frac{d^{2} f}{d z^{2}}\right)+\frac{1}{k}\left(\frac{d k}{d z}\right) \frac{1}{f}\left(\frac{d f}{d z}\right)=\frac{1}{k^{2} r_{0}^{4} f^{4}}-\frac{\varepsilon_{1}}{\varepsilon_{0}^{\prime}} \frac{1}{r_{0}^{2}} .
$$

Transforming the coordinate $z$ and the initial beamwidth $r_{0}$ to dimensionless forms: $\xi=\left(z c / r_{0}^{2} \omega\right)$ and $\rho_{0}=\left(r_{0} \omega / c\right)$, the parameter $\left(r_{0}^{2} \omega / c\right)$ is known as the Rayleigh length. We get the characteristic beam propagation equation for inhomogeneous plasma as

$$
\frac{d^{2} f}{d \xi^{2}}=\frac{\omega^{2}}{c^{2} k^{2} f^{3}}-\frac{1}{k}\left(\frac{d k}{d \xi}\right)\left(\frac{d f}{d \xi}\right)-\frac{\varepsilon_{1}(f)}{\varepsilon_{0}^{\prime}(f)} \rho_{0}^{2} f .
$$

The first term on the right-hand side of (23) is due to the diffraction effect, the second term is due to the plasma inhomogeneities, and the last term is the nonlinear term that is responsible for laser relativistic self-focusing. At $z=0$ ( $f=$ 1 ), for $d^{2} f / d \xi^{2}$ to vanish at some values of $f$ or $\xi$ requires

$$
\rho_{0}^{2}=\frac{4 \omega^{2}}{\omega_{p}^{2}} \frac{\gamma_{0}^{\prime 3}}{q_{0}^{2}}\left[1-\frac{c^{2}}{r_{0}^{2} \omega_{p}^{2}} \frac{\left(8+q_{0}^{2}\right)}{\gamma_{0}^{\prime}}\right] .
$$

Equation (24) expresses the dimensionless beam width $\rho_{0}$ (at $f=1$ ) as a function of $q_{0}$. The function can be drawn as a curve in the $\left(q_{0}, 1 / \rho_{0}\right)$ plane and is generally regarded as the critical power curve.

\section{Density Profiles}

Inhomogeneous plasma is a medium in which the charge density is nonuniform in space. The charge density variation is complex and depends on the type of plasma. In axial inhomogeneous plasma, the electron density changes along axial direction ( $z$-axis) only. For such type of inhomogeneity, the electron density is written as

$$
n(\xi)=n(0) R(\xi)
$$

where $n(0)$ is the density of plasma at $z=0$ and $R(\xi)$ is the density profile function dependent on $z$-coordinate only. Some functions for axial inhomogeneity which have practical importance are as follows:

linear profile:

$$
R(\xi)=1+B \xi
$$

parabolic profile:

$$
R(\xi)=1+(B \xi)^{2}
$$

exponential profile:

$$
R(\xi)=\exp (B \xi)
$$

here $B$ is a constant which determines the slope and is adjustable. Considering the propagation equation (23) of laser beam in a self-created plasma channel, modified plasma frequency can be rewritten as

$$
\omega_{p R}^{2}=\omega_{p 0}^{2} R(\xi) .
$$

Therefore, in presence of density profiles the dielectric function, propagation equation, and beam radius get modified as

$$
\begin{gathered}
\varepsilon_{0 R}^{\prime}=1-\frac{\omega_{p R}^{2} / \omega^{2}}{\gamma_{0}^{\prime}}\left(1-\frac{c^{2}}{\omega_{p R}^{2} r_{0}^{2} f^{2}} \frac{q_{0}^{2} / f^{2}}{\gamma_{0}^{\prime}}\right), \\
\varepsilon_{1 R}=\frac{\omega_{p R}^{2}}{4 \omega^{2}} \frac{q_{0}^{2} / f^{4}}{\gamma_{0}^{\prime 3}}\left(1+\frac{c^{2}}{\omega_{p R}^{2} r_{0}^{2} f^{2}} \frac{8+q_{0}^{2} / f^{2}}{\gamma_{0}^{\prime}}\right), \\
\frac{d^{2} f}{d \xi^{2}}=\frac{\omega^{2}}{c^{2} k^{2} f^{3}}-\frac{1}{k}\left(\frac{d k}{d \xi}\right)\left(\frac{d f}{d \xi}\right)-\frac{\varepsilon_{1 R}(f)}{\varepsilon_{0 R}^{\prime}(f)} \rho_{0}^{2} f, \\
\rho_{0 R}^{2}=\frac{4 \omega^{2}}{\omega_{p R}^{2}} \frac{\gamma_{0}^{\prime 3}}{q_{0}^{2}}\left[1-\frac{c^{2}}{r_{0}^{2} \omega_{p R}^{2}} \frac{\left(8+q_{0}^{2}\right)}{\gamma_{0}^{\prime}}\right] .
\end{gathered}
$$

After initial focusing of the laser, the relativistic mass effect will be much more pronounced in the region of increasing plasma density. Therefore, the laser focuses more during propagation in a plasma density profile. On the other hand, as the equilibrium electron density is an increasing function of the distance of propagation of the laser, the plasma dielectric constant decreases rapidly as the beam penetrates deeper and deeper into the plasma. Consequently, the upward plasma density profile plays an important role in enhancing laser focusing and the laser is more focused in comparison to the uniform distribution of plasma.

\section{Results and Discussion}

Figure 1 represents the variation of the electron density $n_{e}$ with dimensionless distance of propagation $\xi$, for different density profiles correspond to different values of initial electron density and slop parameter $B$. The variation in the electron density of the plasma is partly responsible for the variation of the dielectric behavior along the axial direction. As these density profiles influence the dielectric behavior and nonlinearity of the plasma, it also affects the propagation of the beam in plasma. Here we have considered "linear," "parabolic," and "exponential" increasing density profile plasma. It is evident from Figure 1 that as compared to linear and parabolic profile exponential profile grows rapidly. In case of linear and parabolic profile initially linear profile grows faster as that of parabolic profile but after a certain value of distance of propagation parabolic profile grows faster as that of linear profile density plasma.

The effects of different types of density profiles on the focusing of the beam are illustrated through the critical curves (a plot of initial beam width $\left(\rho_{0}\right)$ against the dimensionless axial irradiance $\left(q_{0}\right)$ shown in Figure 2$)$. On account of different density profiles, namely, without any density, linear, parabolic, and exponential density profiles, initially critical curves decrease and after a certain value the critical curves tend to correspondingly rise. If the initial value of $q_{0}$ 


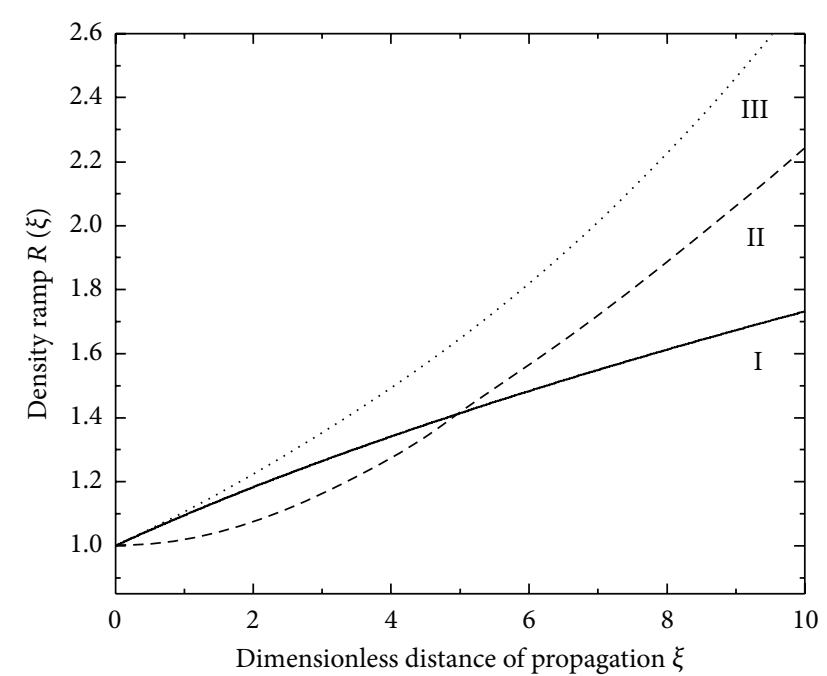

FIgURE 1: Plots for density $\operatorname{ramp} R(\xi)$ with the dimensionless distance of propagation $\xi$ for different types of axial inhomogeneous plasma. Here $B=0.2$. Curves I, II, and III correspond to linear, parabolic, and exponential density profiles, respectively.

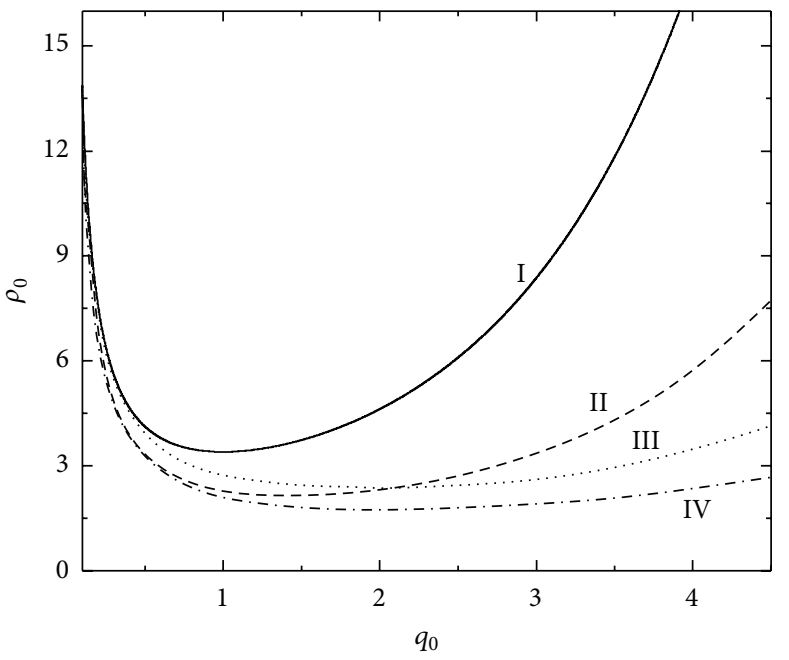

Figure 2: Critical curves, that is, the relation between $\rho_{0}=\left(r_{0} \omega_{0} / c\right)$ and $q_{0}$. Here $B=0.2$. Curves I, II, III, and IV correspond to without any density profile, linear, parabolic, and exponential density profiles, respectively.

and $\rho_{0}$ of a laser beam is such that the point $\left(q_{0}, \rho_{0}\right)$ lies on the critical curve, the value of $\left(d^{2} f / d \xi^{2}\right)$ will vanish at $\xi=0(z=0)$ since the initial value of $(d f / d \xi)$ (in case the wave front in plasma) is zero; the value of $(d f / d \xi)$ continues to be zero as the beam propagates through the plasma. Hence, the initial value of " $f$," which is unity (at $z$ or $\xi=0$ ), will remain unchanged. Thus, the beam propagates without any change in its beam width. Such propagation is known as uniform waveguide propagation. For initial point $\left(q_{0}, \rho_{0}\right)$ of the beam lying above the critical curve, that is, on the opposite side of the curve as the origin $\left(d^{2} f / d \xi^{2}\right)>0$ and for the points lying on the other side, $\left(d^{2} f / d \xi^{2}\right)<0$, therefore, for

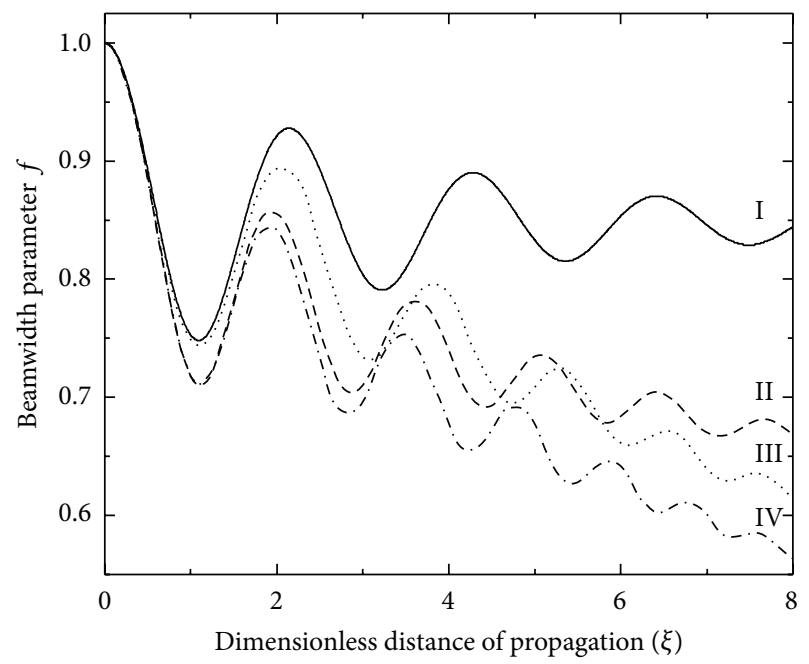

FIGURE 3: Variation of beamwidth parameter $f$ with dimensionless distance of propagation $\xi$, corresponding to $\left(q_{0}, \rho_{0}\right)=(3,2)$ and $B=$ 0.2 . Curves I, II, III, and IV correspond to without any density ramp linear, parabolic, and exponential density profiles, respectively.

the initial point not lying on the critical curve, the beam width parameter will either decrease (for point above) or increase (for point below), as the beam propagates.

Figure 3 shows variation of beamwidth parameter $(f)$ with dimensionless distance of propagation $(\xi)$ corresponding to parameters as mentioned in the figures. In the region of low plasma density, the electrons are expelled from the highintensity region by a ponderomotive force; the nonlinearity in plasma comes by electron mass variation, which is due to intense laser and change in electron density. If there is no density profile, the beamwidth parameter decreases because of the nonlinear effects. As the diffraction effect becomes predominant the beamwidth parameter increases after attaining a minimum value and the laser beam starts diverging owing to saturation of nonlinearity. Hence, the laser becomes focused and defocused and shows an oscillatory behavior. If there is an increasing density profile, the beamwidth parameter decreases up to a Rayleigh length and does not increase dimensionless beamwidth parameter for the second and higher orders decreases continuously, as compared to homogeneous plasma.

Careful observation of these results from Figure 3 indicates that for linearly, parabolic, and exponential axially increasing inhomogeneous density profile plasma, minimum value of the normalized beamwidth parameter $\left(f_{\min }\right)$ for the second and higher orders decreases continuously, as compared to without any density profile inhomogeneous plasma. This is because on increasing electron density, nonlinearity increases. Hence refraction dominates over the diffraction effect, which yields less value of $\left(f_{\min }\right)$. These curves also indicate that for axial distance, exponentially increasing density profile plasma has low value of $\left(f_{\min }\right)$ as compared to the value obtained for without density profile, linear density profile, and parabolically increasing density profile plasma. If linear and parabolic density profile curves are observed, 


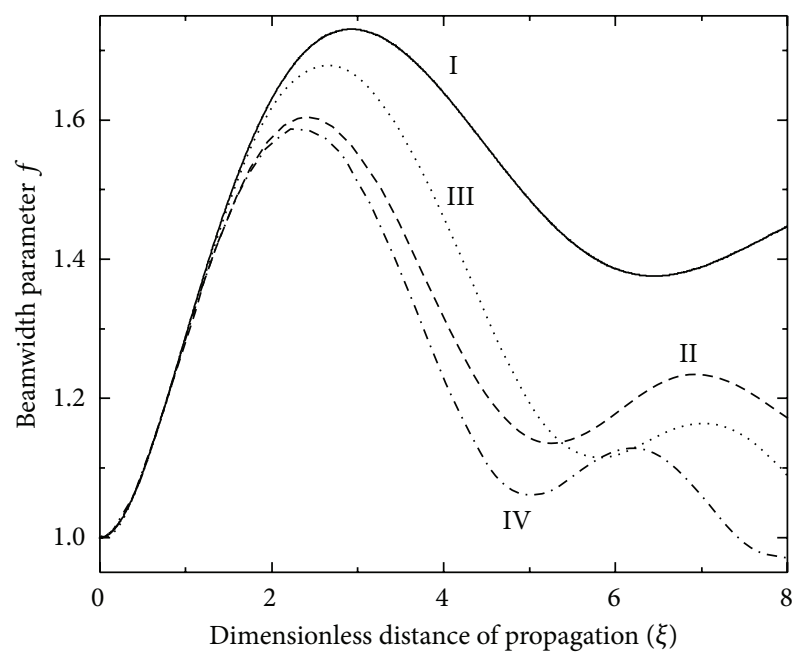

FIGURE 4: Variation of beamwidth parameter $f$ with dimensionless distance of propagation $\xi$, corresponding to $\left(q_{0}, \rho_{0}\right)=(2,1)$ and $B=$ 0.2 . Curves I, II, III, and IV correspond to without any density ramp linear, parabolic, and exponential density profiles, respectively.

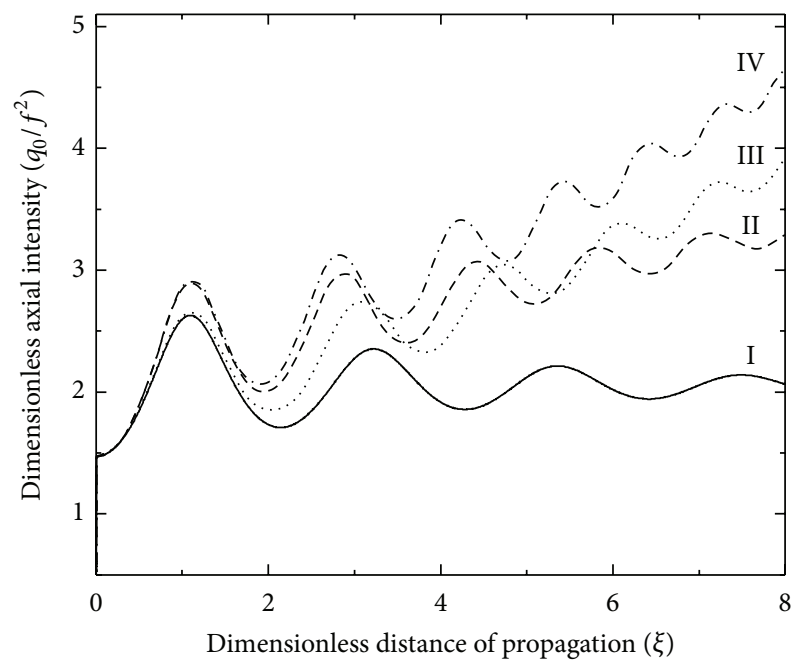

Figure 5: Variation of dimensionless axial intensity $\left(q_{0} / f^{2}\right)$ of the beam with dimensionless distance of propagation $\xi$ corresponding to $\left(q_{0}, \rho_{0}\right)=(3,2)$ and $B=0.2$. Curves I, II, III, and IV correspond to without any density profile linear, parabolic, and exponential density profiles, respectively.

then we find that initially linear density profile curve gives low value of $\left(f_{\min }\right)$ as compared to parabolic density profile plasma but when the value of axial distance (dimensionless distance of propagation $\xi$ ) is more than a certain characteristic length, the parabolically increasing density profile plasma is more effective than linearly increasing density profile plasma. This is due to the fact that for axial distance $\xi$ to be less than the characteristic length, the variation of electron density with $\xi$ is more effective for linearly increasing electron density profile. Similarly we get the results for oscillatory divergence of the beam as shown in Figure 4.
Figure 5 shows the variation of the dimensionless axial intensity of the beam as a function of the dimensionless distance of propagation $\xi$ for inhomogeneous plasma corresponding to the parameters as indicated in the figure. It shows that the successive intensity peaks are higher and the peaks for the exponential variation of inhomogeneity grow faster than those for the linear and parabolic case. These peaks are corresponding to the foci of Figure 3. One may conclude that the successive foci have smaller beam width. The figure also shows that the $\xi$ interval between successive peaks keeps decreasing.

The experimental results on relativistic self-focusing and channel formation have been observed by many authors $[17,18]$. They studied the propagation of an intense laser beam in plasma, including the relativistic and ponderomotive effects. Their results show that the laser beam can acquire a minimum spot size due to the relativistic self-focusing in plasma. Substantially, the analytical and numerical work presented here shows reduction in defocusing effect with the introduction of plasma density profiles. Thus, density profile functions significantly increase relativistic self-focusing of a laser beam in plasma producing ultrahigh laser irradiance over distances much greater than the Rayleigh length which can be used for various applications towards plasma-based accelerators and inertial confinement fusion.

\section{Conflict of Interests}

As the authors of the paper, do not have a direct financial relation that might lead to a conflict of interest for any of the authors. The authors declare that there is no conflict of interests regarding the publication of this paper.

\section{References}

[1] A. Pukhov, Z.-M. Sheng, and J. Meyer-ter-Vehn, "Particle acceleration in relativistic laser channels," Physics of Plasmas, vol. 6, no. 7, pp. 2847-2854, 1999.

[2] P. Sprangle, B. Hafizi, J. R. Peñano et al., "Wakefield generation and $\mathrm{GeV}$ acceleration in tapered plasma channels," Physical Review E-Statistical, Nonlinear, and Soft Matter Physics, vol. 63, no. 5, Article ID 056405, 2001.

[3] E. Esarey, A. Ting, P. Sprangle, D. Umstadter, and X. Liu, "Nonlinear analysis of relativistic harmonic generation by intense lasers in plasmas," IEEE Transactions on Plasma Science, vol. 21, no. 1, pp. 95-104, 1993.

[4] W. Yu, M. Y. Yu, J. Zhang, and Z. Xu, "Harmonic generation by relativistic electrons during irradiance of a solid target by a short-pulse ultraintense laser," Physical Review E-Statistical Physics, Plasmas, Fluids, and Related Interdisciplinary Topics, vol. 57, no. 3, pp. R2531-R2534, 1998.

[5] B. R. Benware, C. D. Macchietto, C. H. Moreno, and J. J. Rocca, "Demonstration of a high average power tabletop soft X-ray laser," Physical Review Letters, vol. 81, no. 26, pp. 5804-5807, 1998.

[6] M. V. Asthana, A. Giulietti, D. Giulietti, L. A. Gizzi, and M. S. Sodha, "Nonlinear propagation of intense short pulses through underdense plasmas," Physica Scripta, vol. T84, article 191, 2000.

[7] S. P. Regan, D. K. Bradley, A. V. Chirokikh et al., "Laser-plasma interactions in long-scale-length plasmas under direct-drive 
National Ignition Facility conditions," Physics of Plasmas, vol. 6, no. 5, pp. 2072-2080, 1999.

[8] C. Ren, B. J. Duda, R. G. Hemker et al., "Compressing and focusing a short laser pulse by a thin plasma lens," Physical Review E-Statistical, Nonlinear, and Soft Matter Physics, vol. 63, no. 2, Article ID 026411, 2001.

[9] P. Jha, R. K. Mishra, A. K. Upadhyaya, and G. Raj, "Self-focusing of intense laser beam in magnetized plasma," Physics of Plasmas, vol. 13, no. 10, Article ID 103102, 2006.

[10] D. N. Gupta and H. Suk, "Enhanced focusing of laser beams in semiconductor plasmas," Journal of Applied Physics, vol. 101, Article ID 043109, 2007.

[11] M. A. Varshney, B. Rathore, S. Sen, and D. Varshney, "Propagation modes and regimes of intense laser beam in magnetized plasma," Journal of Modern Optics, vol. 56, no. 21, pp. 2368-2376, 2009.

[12] S. Sen, B. Rathore, M. Varshney, and D. Varshney, "Nonlinear propagation of intense electromagnetic beams with plasma density ramp functions," Journal of Physics: Conference Series, vol. 208, Article ID 012088, 2010.

[13] M. A. Varshney, S. Shukla, S. Sen, and D. Varshney, "Relativistic cross-focusing of extraordinary and ordinary modes in a magnetoactive plasma," Journal of Plasma Physics, vol. 79, no. 5, pp. 953-961, 2013.

[14] S. Sen, M. A. Varshney, and D. Varshney, "Relativistic propagation of linearly/circularly polarized laser radiation in plasmas," ISRN Optics, vol. 2013, Article ID 642617, 8 pages, 2013.

[15] S. A. Akhmanov, A. P. Sukhorukov, and R. V. Khokhlov, "Selffocusing and diffraction of light in a nonlinear medium," Soviet Physics Uspekhi, vol. 10, no. 5, article 609, 1968.

[16] M. A. Varshney, S. Sen, B. Rathore, and D. Varshney, "Propagation regimes of intense circularly polarized laser beam in magnetoactive plasma," Optik, vol. 122, no. 5, pp. 395-401, 2011.

[17] R. Fedosejevs, X. F. Wang, and G. D. Tsakiris, "Onset of relativistic self-focusing in high density gas jet targets," Physical Review E-Statistical Physics, Plasmas, Fluids, and Related Interdisciplinary Topics, vol. 56, no. 4, pp. 4615-4639, 1997.

[18] B. Hafizi, A. Ting, P. Sprangle, and R. F. Hubbard, "Relativistic focusing and ponderomotive channeling of intense laser beams," Physical Review E-Statistical Physics, Plasmas, Fluids, and Related Interdisciplinary Topics, vol. 62, no. 3, pp. 4120-4125, 2000 . 

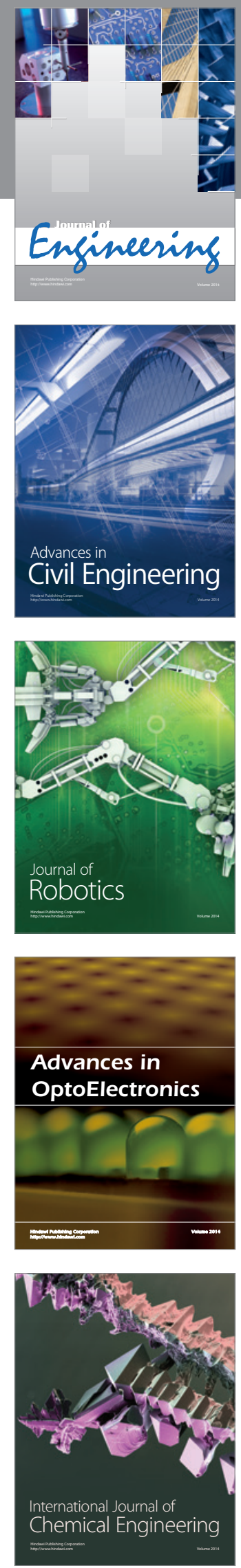

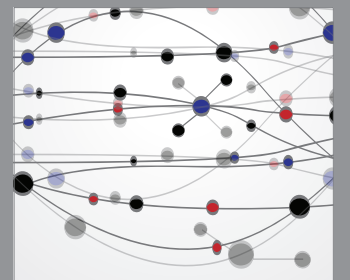

The Scientific World Journal
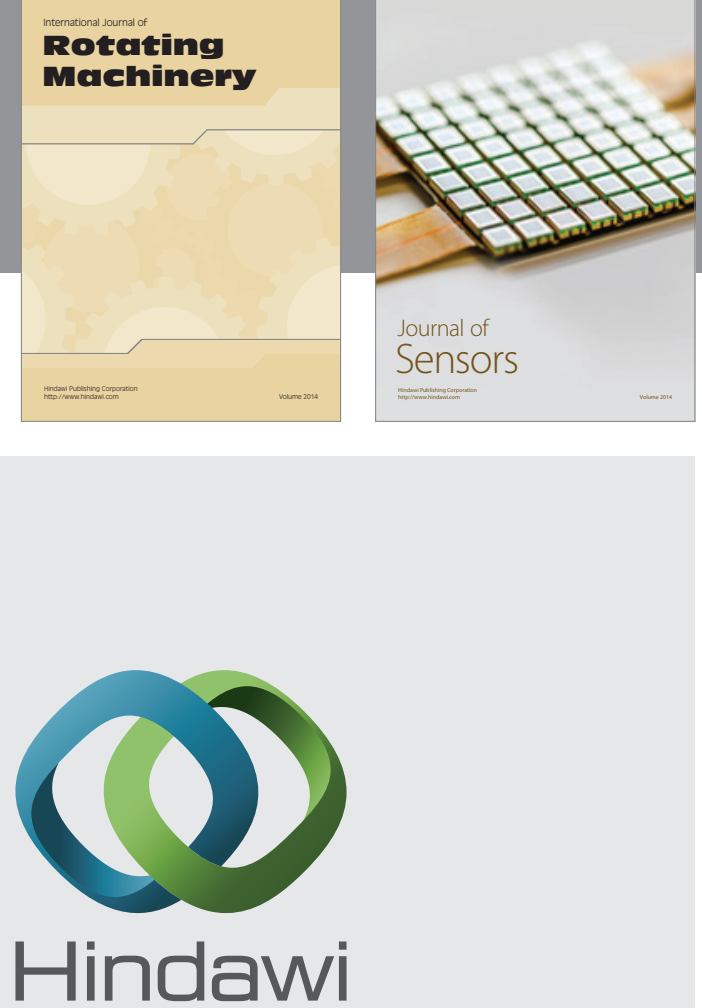

Submit your manuscripts at http://www.hindawi.com
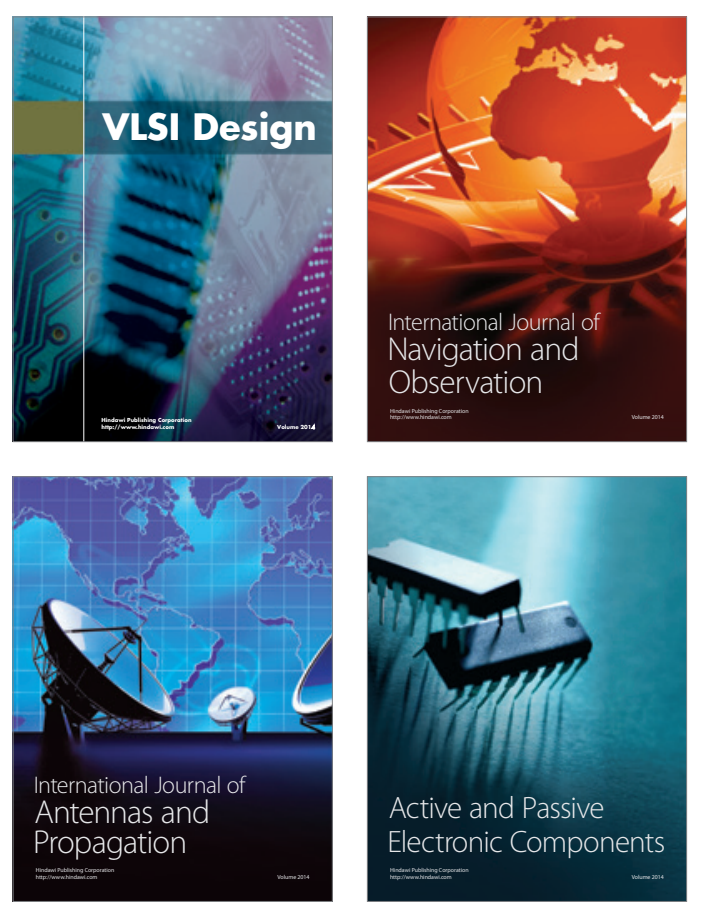
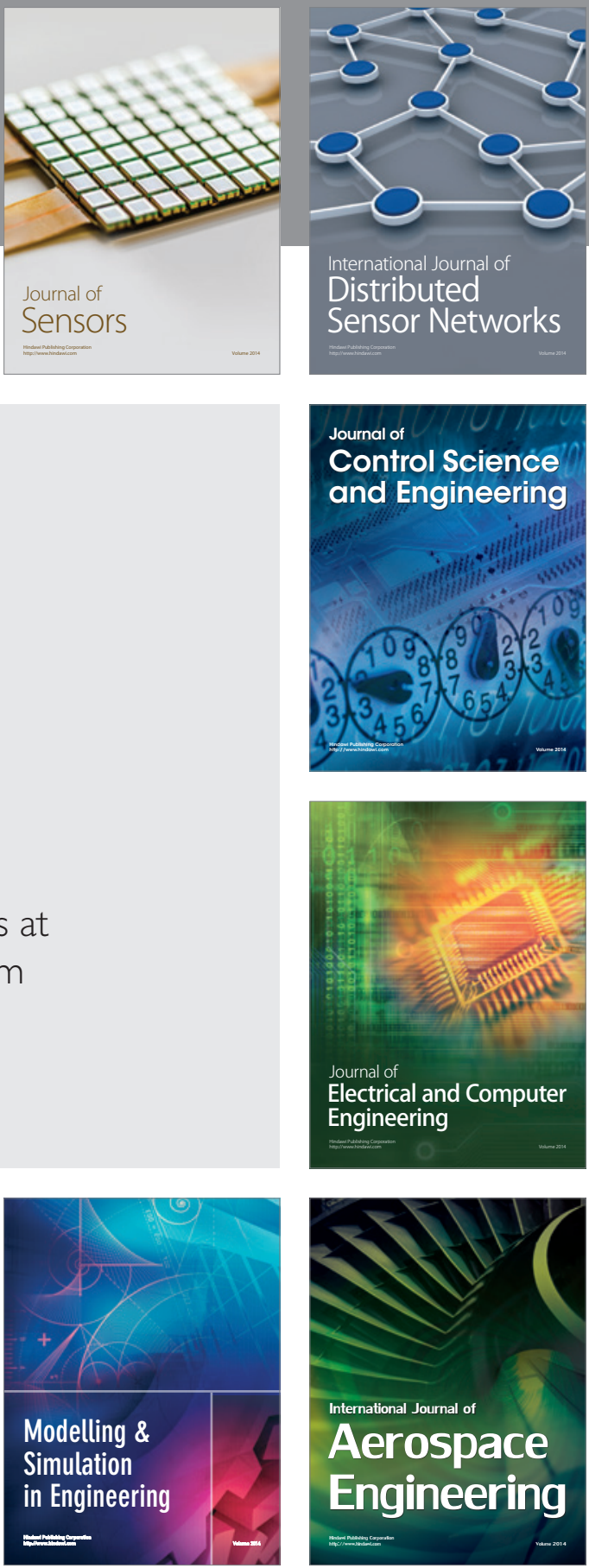

Journal of

Control Science

and Engineering
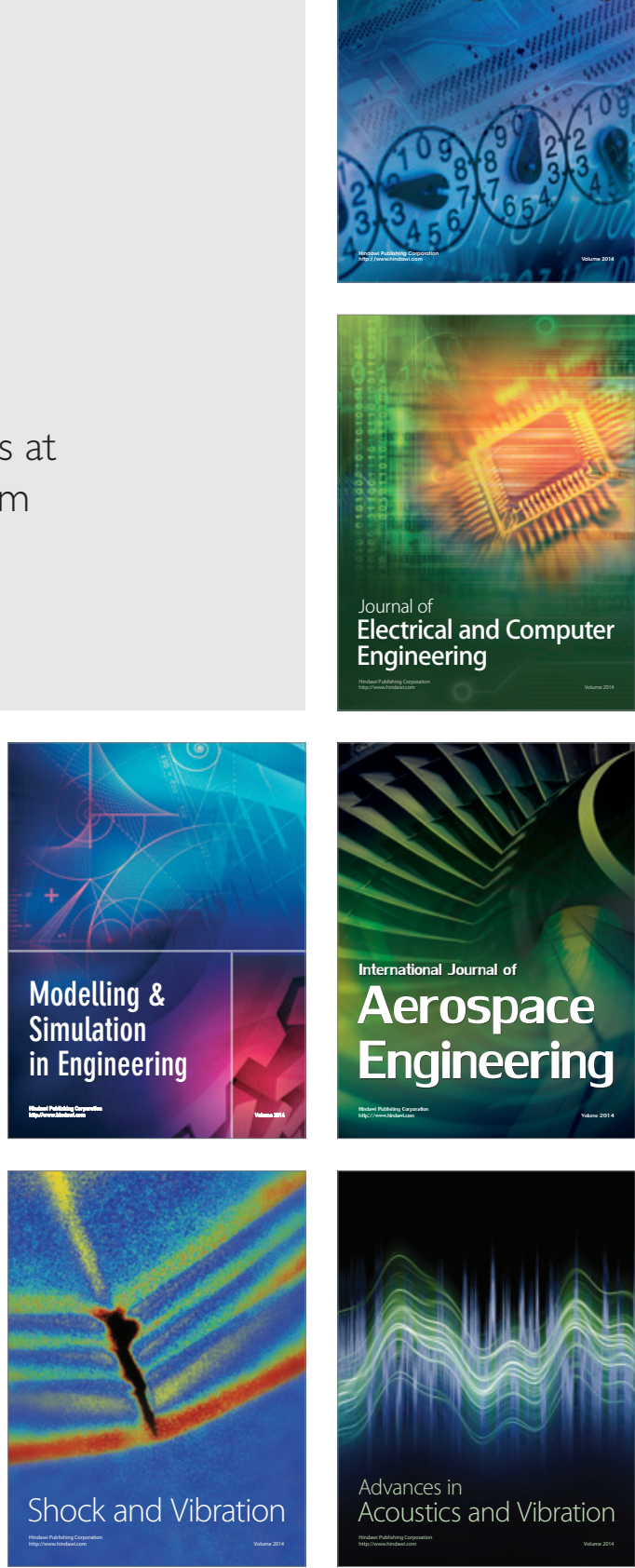